\title{
MODELLING EMS MAGLEV SYSTEMS TO DEVELOP CONTROL ALGORITHMS
}

\author{
Victor Amoskov \\ JSC “NIIEFA”, Russia \\ amoskov-sci@yandex.ru
}

\author{
Daria Arslanova \\ JSC "NIIEFA", Russia \\ arslanova-sci@yandex.ru
}

\author{
Gennady Baranov \\ JSC "NIIEFA", Russia \\ baranov@sintez.niiefa.spb.ru
}

\begin{abstract}
Alexandr Bazarov
JSC "NIIEFA", Russia

bazarov-sci@yandex.ru
\end{abstract}

Valery Belyakov

Saint Petersburg State University, Russia belyakov@sintez.niiefa.spb.ru

\author{
Alexey Firsov \\ JSC “NIIEFA", Russia \\ firsov-sci@yandex.ru
}

\section{Marina Kaparkova JSC “NIIEFA", Russia kaparkova-sci@yandex.ru}

Vladimir Kukhtin

JSC “NIIEFA", Russia

kukhtin-sci@yandex.ru

\author{
Andrey Kavin \\ JSC "NIIEFA", Russia \\ kavina@sintez.niiefa.spb.ru
}

\section{Vladimir Kuzmenkov \\ JSC "NIIEFA", Russia}

kuzmenkov@sintez.niiefa.spb.ru

\author{
Mikhail Khokhlov \\ JSC “NIIEFA", Russia \\ khokhlov@sintez.niiefa.spb.ru
}

\author{
Eugeny Lamzin \\ JSC “NIIEFA", Russia \\ lamzin-sci@yandex.ru \\ Andrei Lantzetov \\ JSC "NIIEFA", Russia \\ lancetov@sintez.niiefa.spb.ru
}

\author{
Alexey Labusov \\ JSC "NIIEFA", Russia \\ labusov@sintez.niiefa.spb.ru
}

\begin{abstract}
Andrey Nezhentzev
JSC “NIIEFA”, Russia nezhentzev-sci@yandex.ru
\end{abstract}

\author{
Mikhail Larionov \\ JSC “NIIEFA", Russia \\ larionov-sci@yandex.ru
}

\author{
Alexandr Ovsyannikov \\ Saint Petersburg State University, Russia \\ ovc74@mail.ru
}

\author{
Igor Rodin \\ JSC "NIIEFA", Russia \\ rodin-sci@yandex.ru \\ Nikolay Shatil \\ JSC "NIIEFA", Russia \\ shatil@sintez.niiefa.spb.ru
}

\author{
Sergey Sytchevsky \\ Saint Petersburg State University, Russia \\ sytch-sie@yandex.ru
}

\section{Vyacheslav Vasiliev \\ JSC “NIIEFA", Russia \\ vasiliev-sci@yandex.ru}

Elena Zapretilina

JSC "NIIEFA", Russia

zapretilina@sintez.niiefa.spb.ru

Article history:

Received 15.04.2018, Accepted 15.05.2018

\begin{abstract}
Electromagnetic suspension (EMS) system for magnetically levitated vehicles can utilize different types of magnets, such as room temperature electromagnets,
\end{abstract}

superconducting magnets as well as permanent magnets. In the course of the study the trichotomy has been applied to the electromagnetic suspension system. The EMS configuration considered in this paper has been treated as a combination of these three types of magnets modelled individually. Results of computations were compared to measurements on a working prototype that 
provided stable levitation of a platform weighing above $190 \mathrm{~kg}$. A good agreement between the simulated and measured parameters enabled verification of the computational models for separate magnets, selection of efficient control algorithms for a combined EMS system, validation of numerical procedures for payload scaling for practical maglev applications. The combined EMS under study has demonstrated improved power consumption as compared to the conventional EMS. Optimal control algorithms for a combined EMS should factor in various criteria, including rapidity, stability, power consumption, weight, reliability, etc. Different types of magnets can be integrated into a single module to reach the desired performance. Hence, the optimum solution for the EMS design and relevant control algorithms should be searched within a common procedure using detailed computational models.

\section{Key words}

Maglev, hybrid system, simulation, control algorithm, computational technique, magnetic field.

\section{Introduction}

The magnetic levitation technology is principally categorized [Zhuravlyov, 2003; Zhigang at.al., 2015; Lee et.al., 2006; Yan, 2006; Yan, 2008] as electromagnetic suspension (EMS), or attractive levitation, and electrodynamic suspension (EDS) [Bocharov, Nagorsky, 1991; Dzenzersky et.al., 2001; Kim, 2007], or repulsive levitation. Each type offers its advantages at different speed ranges and operating modes. The magnetic field to levitate a vehicle can be provided by either normal conducting electromagnets (EM), or superconducting (SC) magnets, or high-coercivity permanent magnets (PM) [Mayer and Meins, 1984; Qiang et.al. 2017]. With the technology advancing in superconductors and magnetic materials, hybrid magnet systems become promising for commercial application.

The search for the most efficient suspension systems for the maglev transport leads to configurations that employ different types of magnets [Morishita et.al., 1989]. Magnets synergy results in better performance and lower energy consumption.

At the first stage of our study the electromagnetic suspension has been trichotomized into a combination of three types of magnets, EM, SC, and PM, modelled individually. Then, individual magnet models are integrated into a combined EMS system.

Results of computations with the individual models have been compared to measurements on a special measurement stand. Simulations with integrated models have been compared to measurements on a working prototype capable of stable levitation of a platform weighing more than $190 \mathrm{~kg}$.

A good agreement between the simulated and measured parameters enables (1) verification of the compu- tational models for individual magnets; (2) selection of efficient control algorithms for the combined EMS system, (3) validation of numerical procedures for payload scaling for practical maglev applications.

The combined EMS under study has demonstrated improved power consumption as compared to a conventional EMS utilizing room temperature EM only.

The urge towards improved controllability and energy consumption has motivated authors to develop and study a series of possible configurations of maglev systems [Patent 2573524] that includes:

- combined EMS-EDS system efficient over the entire speed range;

- EM-SC-PM EMS system utilizing normal conducting coils, hot temperature superconducting (HTS) coils and $\mathrm{Nd} \mathrm{Fe} \mathrm{B}$ permanent magnets;

- several configurations of the track and onboard magnets for EDS systems.

The basic steps to proceed to their practical implementation are:

- engineering design of all types of magnets and production/procurement pre-qialification;

- construction of detailed computational models to accurately describe spatial distribution of magnetic forces in terms of their levitation, drag, and guidance components, heat release in conducting structures, and external fields;

- development of control algorithms for nominal, transient and fault operation.

An original computational technique created for EM simulations of accelerators and tokamak magnets was adapted [Amoskov et.al., 2008; Belov et.al., 2008] to the maglev application [Amoskov et.al., 2014; Amoskov et.al., 2015a; Amoskov et.al., 2015b; Amoskov et.al., 2016a]. The modified technique enables exact evaluation of required magnetic parameters, as well as analysis, synthesis and optimization of the suspension system design.

Theoretical and numerical analyses were accomplished for a number of suspension system configurations. A series of models was developed, designed and manufactured by the JSC "NIIEFA" in collaboration with the St.Petersburg State University to verify experimentally the design solutions and simulation accuracy.

An essential issue to guarantee operability of the EMS systems is the development of reliable control algorithms. The maglev vehicle movement differs greatly from that of conventional trains and is inherently unstable thus necessitating particular tools to analyze and control. The results obtained in our study have reasserted the conclusion of fundamental works [Bocharov, Nagorsky, 1991; Dzenzersky et.al., 2001; Kim, 2007] that sufficiently robust and stable control algorithms cannot be created without the use of detailed 3D models of electromagnetic systems. This involves building 3D computational models of the magnets and 
track structures as well as allowing for non-linear properties of magnetic materials.

In previous studies the authors have analyzed operational parameters of several EDS configurations.

Their findings, in particular, confirm the minimum speed criterion (see, e.g., [Zhuravlyov, 2003; Kim, 2007]) that enables levitation (for example, above 80 $\mathrm{km} / \mathrm{h}$, as estimated in [Zhuravlyov, 2003]). Therefore, the EDS application is ranged within the long-distance and high-speed transportation. In contrast, the EMS maglev trains can levitate at a stop for loading or boarding as well as at the low speed typical for urban transport.

Control algorithms for a combined EMS [Li et.al., 2005] should be optimized with various physical criteria in mind, such as rapidity, stability, power consumption, weight, reliability, safety etc. Different types of magnets can be integrated into a single structural module [Morishita et.al., 1989] to achieve enhanced performance with their synergism. Hence, the optimum solution for the EMS design and relevant control algorithms should be searched within a common procedure using detailed computational models.

The purpose of this study is to analyze components of the EMS system [Bocharov, Nagorsky, 1991; Dzenzersky et.al., 2001; Kim, 2007] to proceed to development of efficient control algorithms for magnetically levitated levitation transport [Patent 2573524].

\section{Combined EMS System}

EMS acts as an active magnetic support [Zhuravlyov, 2003] that uses attraction between ferromagnetic guideways and magnets installed on a levitation frame wrapping the track. The attracting force overcomes the gravitational force thus levitating the vehicle on the track. To stabilize the suspension an active control system is used that adjusts the magnetic induction in the levitation gap and thus the attractive forces of the magnets. The simplest schematics is illustrated in Fig. 1 (see Fig. 1.8 in [Zhuravlyov, 2003], which is transformed for subsequent presentation). With the EMS system, the levitation force can be generated and controlled at any propulsion speed and at rest that makes it convenient for many operating conditions on the transport. EMS is used in the static modes (loading, unloading, stop) and at speeds below $400-450 \mathrm{~km} / \mathrm{h}$, which is characterized by relatively low energy consumption.

The combined EMS configuration under study includes:

- a series of onboard room-temperature electromagnets that provide active control;

- an array of permanent magnets to suspend the weight [Patent 2573524];

- HTSC magnets with low current variations used to balance the vehicle by controlled suspension at track turns and load changes.

The theoretical and numerical analysis have been sup-

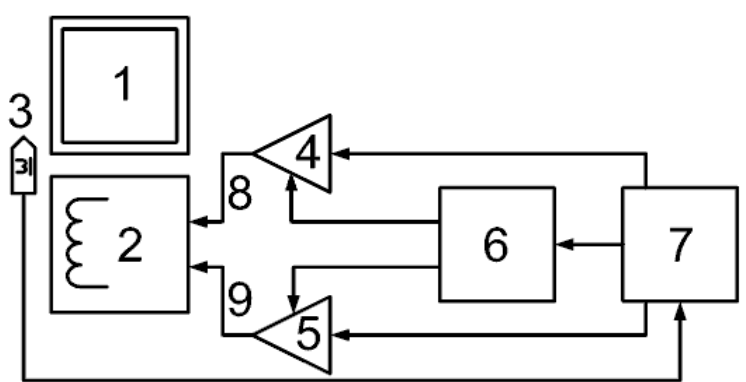

Figure 1. EMS suspension system: 1 - ferromagnetic guideway, 2 - electromagnets, 3 - air gap sensor, 4,5 - power amplifiers, 6 - power source, 7 - controller and DAQ, 8 - supply current, 9 control current.

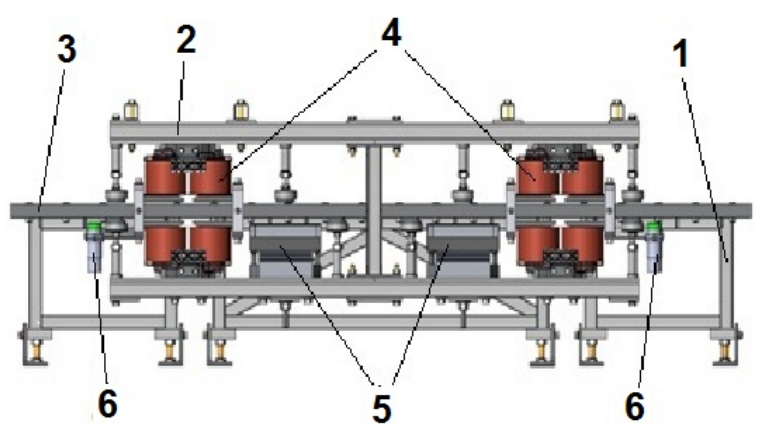

Figure 2. Prototype of combined EMS with room temperature electromagnets and permanent magnets. 1 - guideway structures, 2 suspension frame, 3 - ferromagnetic track, 4 - electromagnets, 5 - PM bearing magnets, 6 - air gap sensor.

ported with the design and optimization of a scaled combined EMS prototype.

In the combined EMS, the lift and drug forces depend non-linearly on the coil current. Ferromagnetics in the elements of magnetic systems such as guideway structures make this effect even more prominent. Note that for computations of EMS the magnetostatic approach is applicable [Tamm, 1989].

Parametric simulations with the special computational technique applied have enabled construction of the scaled prototype capable to reflect adequately the EMS performance. This results in sufficient savings of cost and materials. To imitate a maglev vehicle the best way, the prototype has a realistic levitation gap, response speed and accuracy of the gap control system, and power source characteristics.

The experiments with the prototype were aimed to: - minimization of energy consumption in terms of the specific power $P_{e}$ required to levitate the load of $1 \mathrm{~N}$;

- synthesis of the optimal configuration of EMs capable to create the largest levitation force per unit weight;

- validation of the energy efficiency and environmental safety;

- optimization of technologies used in the manufacture 
of the entire EMS system and its components;

- testing and verification of the control algorithms;

- adjustment of the gap control system and EM power supply at static and loading modes.

The basic parameters of the combined EMS prototype are as follows:

- levitation gap - $5 \mathrm{~mm}$;

- distance between midlines of the ferromagnetic guideways - $500 \mathrm{~mm}$;

- track cross-sections $-25 \times 50 \mathrm{~mm}$;

- system weight (magnets+bogie) - $120 \mathrm{~kg}$;

- total levitated load - $250 \mathrm{~kg}$ or more.

Prior to modelling and testing, magnetic measurements were performed on samples of all types of steels used in the prototype structures. Special measurement techniques and equipment developed at Efremov Inst. were applied. The measured non-linear variations of magnetic properties were then taken into account in numerical models of the hybrid EMS components. This allowed an optimal selection of the steels as a trade-off between cost, performance, and manufacturing requirements.

\section{Suspension Frame and Guideways}

Basically, the hybrid EMS prototype represents steel guideways with a suspension frame on which the EMS system is mounted (see Fig. 2). Development, manufacture and testing of the frame and track structures for the scaled EMS prototype allowed us to assess requirements for production of full-scale systems and verify numerical methods for scaling.

Simulated spatial distributions of EM forces were used as inputs for a stress-strain analysis of the frametrack system. Mechanical stresses and deformations were investigated for a number of operational conditions. Particularly, the frame rigidity was evaluated with respect to the arrangement of EM mounting areas. Obtained results enabled optimization of the frame design.

\section{Normal Conducting Electromagnets}

The simplest formula to calculate the attractive force of a U-core EM [Popov et.al., 1956] demonstrates that this force depends on the square of the field in the levitation gap and the cross-section area of the poles. Design and fabrication of room temperature electromagnets for the scaled prototype were undertaken with a complete production cycle in mind that implies engineering calculations, high-level design concept with respect to the desired attractive force, detailed design, design optimization; development of technological documentation, manufacture, tests (see Figs. 3, 4).

\section{EM-PM Suspension System}

The EMS system of the scaled prototype used for testing control algorithms comprises normal conduction

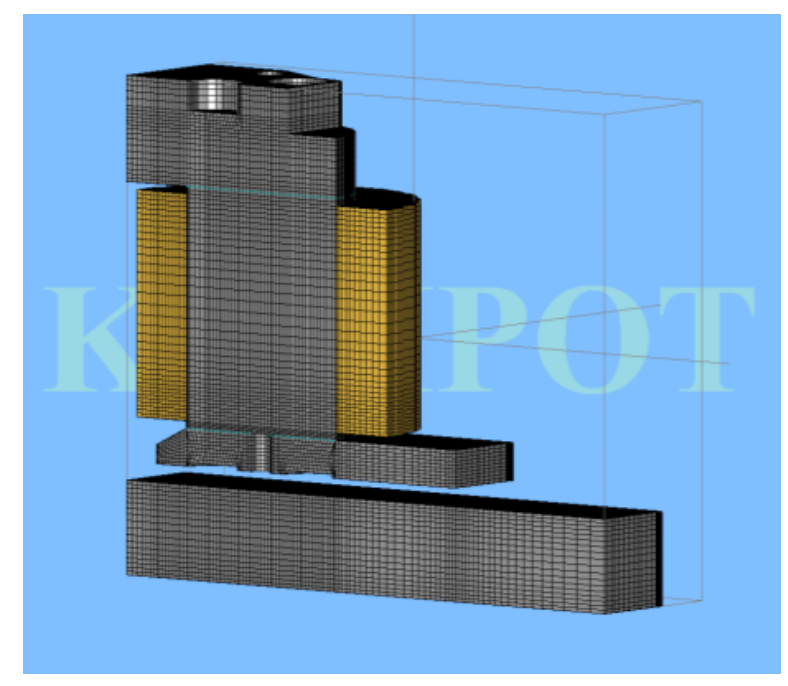

Figure 3. 3D FE model of electromagnet taking into account symmetry. Meshing over air is hidden.

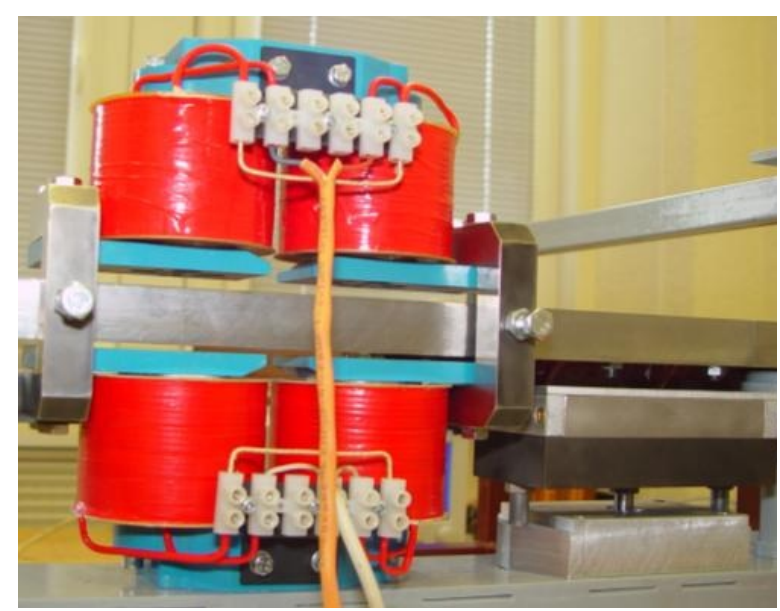

Figure 4. Test bench with control EMs and bearing PMs. Standstill levitation.

EMs combined with SC coils or PMs.

$\mathrm{PMs}$ and SC magnets create the basic levitation force to compensate the weight of a static load. Further on, these magnets will be referred to as the bearing magnets (BM).

The normal conduction EMs are responsible for stable levitation, and their bearing capacity is of minor importance. The electromagnets will hereinafter be referred to as control magnets (CM).

Such configuration allows significant reduction in the energy consumption.

At the first stage the EM-PM hybrid EMS was studied. A challenging issue is development of efficient control algorithms for the different types of magnets employed. Generally, EMs and PMs are located at different distances from the guideways, and the lift force exhibits non-linear variations with the air gap.

Bearing magnets utilizing PMs were developed at the 


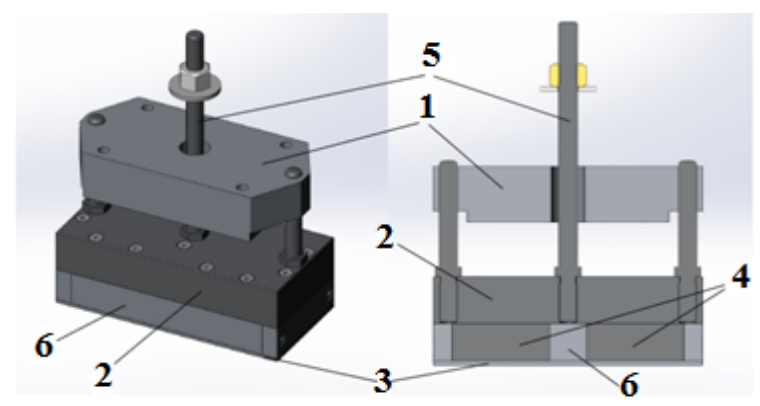

Figure 5. Bearing magnet with PMs array: overview and sectional view. 1 - fixture, 2 - steel magnetic circuit, 3 - PM case base plate, 4 - PM array, 5 - stud pin, 6-PM case.

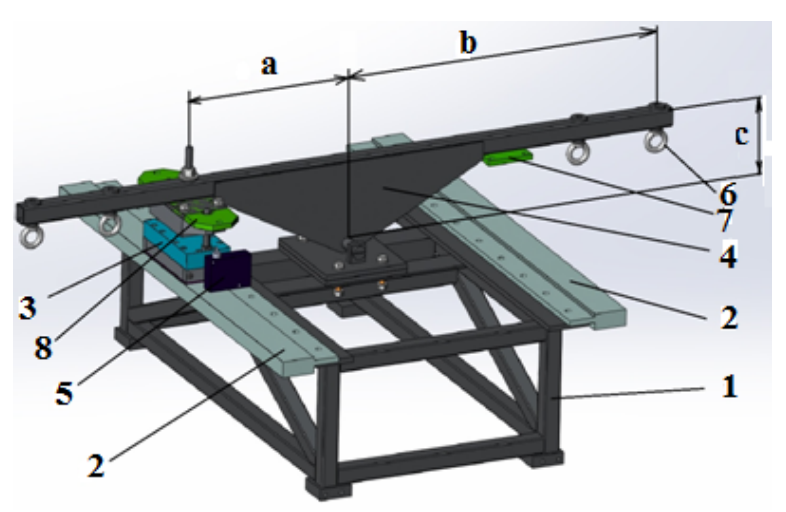

Figure 6. Bearing magnet on test bench to measure attractive force: 1 - guideway structures, 2 - steel rails, 3 bearing magnet with PMs array, 4 - balancing arm, 5 - laser triangulating sensor, 6 compensating load, 7 - EM base plate, 8 - PM base plate.

initial stage of the work. The bearing magnet design is shown in Fig. 5. A comparison of measured data with the calculated results has been done to verify the computational models.

\section{Measurements with PM Suspension Unit}

The bearing magnet installed on a test bench (see Fig. 6) contains a special array of permanent magnets. The test bench imitates the guideway structures (1) with two steel rails (2). The PM unit (3) is mounted on a balancing arm (4) above the rails. The arm axis is collinear with the rails. The PM unit is connected with a tightening screw to a force gage. The air gap between the PM suspension unit and the gyideways is changed with the tightening screw and measured with a laser triangulating sensor (5) with the resolution of $0.09 \mu \mathrm{m}$. As at a low air gap the attractive force is above the scale range of the force gage, a compensating load (6) is attached to the balancing arm.

In the measurements, the arm friction was supposed to be negligibly low and excluded from the observations. With respect to environmental noise (vibrations etc), the air gap measurement uncertainty was evalu-

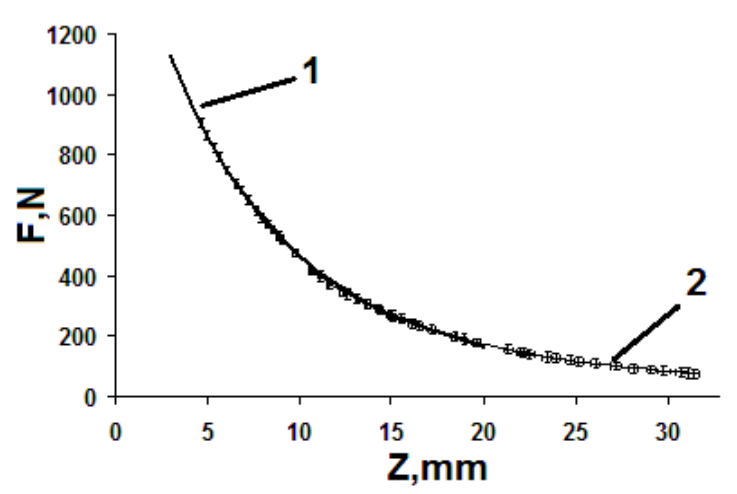

Figure 7. Attractive force: (1) simulated and (2) measured (with estimates of measurement errors).

ated from the readout repeatability as $\pm 5 \mu \mathrm{m}$.

The reference air gap between the PM suspension unit and the guideways was taken as $6 \mathrm{~mm}$. This corresponds to the level position of the balancing arm.

As seen from Fig. 7, the measured data agreed well with the simulated attractive force that verifies the accuracy of the computational model.

Similar measurements were performed with EMs and then compared to simulated data. Again, the computational model was found to give reliable predictions.

The verified computational models of PMs and EMs were used to develop control algorithms for a levitated vehicle (algorithms for position control of levitation system with respect to the ferromagnetic guide track structure). The proposed design concept and control algorithms were confirmed in experiments with the hybrid EM-PM EMS prototype (Fig. 2) that demonstrated stable levitation and desired load capacity.

\section{Hybrid EMS with HTSC Magnets}

HTSC magnets are a promising technology in the maglev transportation since they offer low power consumption. As with the EM-PM EMS model, the development of the Hybrid EMS with HTSC magnets addressed a complete production cycle (Figs. 8, 9).

\section{Conclusions}

A concept is proposed for an EMS system that combines various types of magnets and is suited for the maglev application. The design solutions utilize original techniques and software tools protected by national patent [Patent 2573524].

The virtual prototyping has been effectively applied to mature the design. Parametric simulations with 3D models were used to verify and optimize the configuration and performance of the suspension system in the static mode and movement with low energy consumption. The established computational technique enables reliable scaling of the simulated operational parameters with respect to the practical load capacity and other 


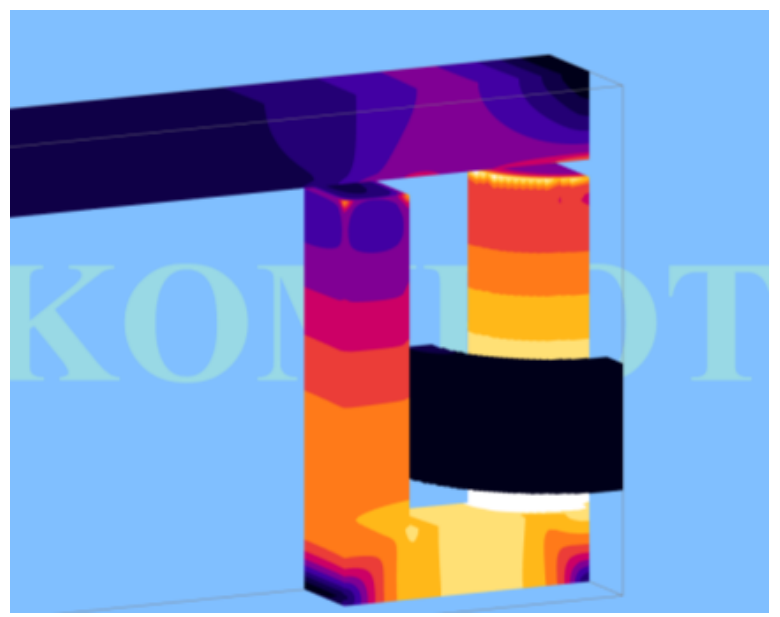

Figure 8. Simulated field map of bearing HTSC magnet (coil and core). $1 / 4$ symmetrical segment.

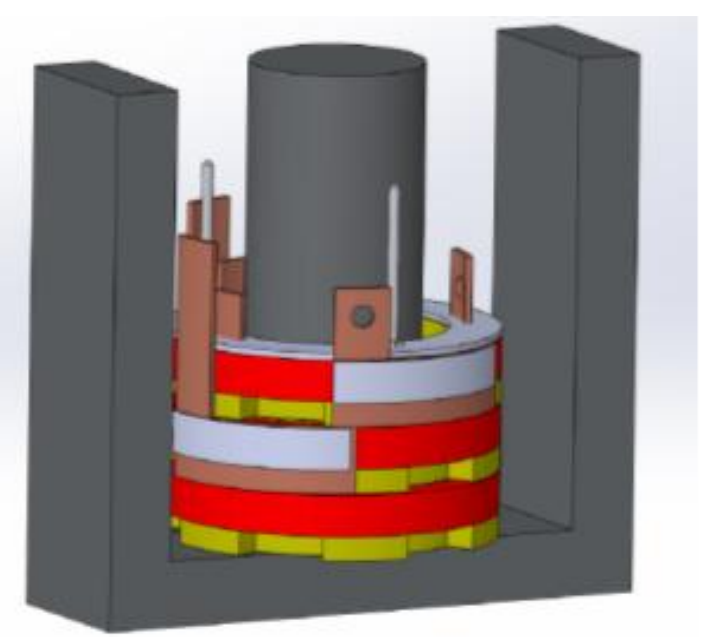

Figure 9. Computational model of bearing HTSC magnet. Overview.

characteristics. The proposed approach provides versatility of levitation system design.

Experiments performed on the test bench and the EMS prototype have demonstrated the accuracy of numerical modelling.

The use of detailed computational models ensures accurate description of the response of the system thus facilitating development of efficient control algorithms. The status information can be automatically obtained from sensors measuring the air gap, the acceleration, the current, ets. Optimal controller parameters are derived from multi-parametric functions of the levitation gap and its derivatives to achieve good robustness and adaptability to system parameter perturbations.

The parametric simulations followed with the construction of physical models and tests make it possible to adjust the production cycle prior to actual manufacture. Such approach provides cost-efficient and flexible development of suspension systems for maglev transport application.

\section{References}

Amoskov, V.M., Belov, A.V., Belyakov, V.A. et.al. (2008) Plasma Devices and Operations. 16(2), pp. 89-103.

Amoskov, V.M., Arslanova, D.N., Bazarov, A.M. et.al. (2014) VESTNIK of St.Petersburg University. Ser. 10 Issue 4, pp. 5-15.

Amoskov, V.M., Arslanova, D.N., Bazarov, A.M. et.al. (2015) VESTNIK of St.Petersburg University. Ser. 10 Issue 2, pp. 8-32.

Amoskov, V.M., Arslanova, D.N., Bazarov, A.M. et.al. (2015) VESTNIK of St.Petersburg University. Ser. 10 Issue 3, pp. 4-21.

Amoskov, V.M., Arslanova, D.N., Bazarov, A.M. et.al. (2016) VESTNIK of St.Petersburg University. Ser. 10 Issue 3, pp. 4-17.

Belov, A.V., Belyakova, T.F., Gornikel, I.V. et.al. (2008) IEEE Transact. on Appl. Supercond, 18(2), pp. 1609-1612.

Bocharov, V., Nagorsky. V. (editors) (1991) Vehicles with magnetic suspension. Mashinostroenie. Moskow.

Dzenzersky, V., Omelyanenko, V., Vasiliev, S., Matin, V., Sergeev, S. (2001) High-speed levitating transport with electrodynamic suspension. Naukova dumka. Kiev.

Kim, K.K. (2007) Transportation systems employing magnetic suspension and superconducting magnet technology. Educational and Methodological Centre for Rail Transport Technology. Moscow.

Lee, H.W., Kim, K.C., and Lee, J.N. (2006) Review of Maglev Train Technologies. IEEE Trans. on Magn., 42(7), pp. 1917-1925.

Li Yungang, Cheng Hu, Zhang Ding (2005) Levitation control system design and analysis of EMS hybrid suspension with electromagnets and permanent magnets. Proceedes in 24th Chinese Control Conference, Guangdong.

Mayer, W.J. and Meins, J. (1984) The use of Electromagnets and Permanent Magnets in Magnetic Levitation Technology. Journal de physique, pp. C1-739C1-745.

Morishita, M. et.al. (1989) A new Maglev system for magnetically levitated carrier system. IEEE Transactions on Vehicular Tech., 38(4), pp. 230-236.

Patent RU 2573524 (2014) A hybrid electromagnetic suspension system for levitated vehicles.

Popov, V.S., Mansurov, N.N, Nikolaev, S.A. (1956) Electrotechnics. Ministry of Municipal engineering Publ. Moscow.

Qiang Chen, Jie Li, Guanchun Li, and Siyang Zhou (2017) Magnetic Flux Density Feedback Control for Permanent Magnetic-Electromagnetic Hybrid Suspension System. Proceeds in ICMME 2016, 95. 
Tamm, I. (1989) Basis of Electricity Theory, Nauka, Moscow.

Yan, L.G. (2006) Progress of the Maglev Transportation in China. IEEE Trans. on Appl. Supercond., 16(2), pp. 1138-1141.

Yan, L.G. (2008) Development and Application of the maglev transportation System. IEEE Trans. on Appl. Supercond., 18(2), pp. 92-99.

Zhigang Liu, Zhiqiang Long, Xiaolong Li (2015) Maglev Trains. Key Underlying Technologies, Springer, London

Zhuravlyov, Y.N. (2003) Magnetic bearings. Theory, calculation, application. Polytechnika. St.Petersburg. 